\title{
GALACTIC WOLF-RAYET DISTRIBUTION AND IMF
}

\author{
BAMBANG HIDAYAT \\ Observatorium Bosscha, ITB \\ Lembang, West Java, Indonesia
}

\begin{abstract}
Wolf-Rayet star to OB star number ratios in some selected regions in the Galaxy are presented. The variations of the number ratio as a function of location is found to be marginal.
\end{abstract}

\section{Introduction}

The WR/OB progenitor number ratio in the Galaxy is the important parameter for the determination of the initial mass of WR progenitors. Observationally, the number ratio is determined from the observed distributions of WR and OB stars in the Galaxy. The observed values are then compared with predicted values derived from theories of stellar evolution. Discrepancies between the two values have been found (e.g., Conti et al., 1983) and thus complicate the exact determination of the WR initial mass. Introduction into the theory of effects of metallicity and convective core overshooting can largely remove the discrepancy.

However, the initial mass function depends critically on the luminosity function, which, in turn, determines the SFR. While in principle the luminosity function can be determined from a volume-limited sample, it must be assured the the co-spatial sample is complete. The purpose of this note is to indicate the sensitivity of the SFR, in view of the limited sample distribution.

\section{The local galactic structure}

Smith (1968) was the first to note a radial variation in the galactic WR subtype distribution. Using larger samples of WR stars with newly calibrated parameters, van der Hucht et al. (1988) and Conti \& Vacca (1990) have found similar effects and confirm that, in general, WR stars delineate the local galactic spiral arms. These studies claim that the WR sample is complete within $2.5 k p c$ - perhaps $3.0 k p c$ - from the Sun.

Within the same volume element OB stars have also been surveyed (e.g. by Garmany, 1982). The distribution of the most massive OB stars does show the same pattern of the commonly accepted local spiral structure, while the less massive stars are distributed in a more uniform way.

Therefore the number densities of the WR and OB stars can readily be calculated, and intercompared.There are, however, some subtle differences that one has to take into account, in order to access the meaning of intercomparison. 
The lack of a 2-dimensional classification for all OB stars excludes the exact determination of the spatial distribution of this type of stars in the Galaxy. Therefore one has to be cautious for a direct comparison of number densities. An other fact is that there is a region which seems to be devoid of WR stars, but still harboring some OB stars. This refers to the direction of $l \approx 245^{\circ}$, in which FitzGerald and Moffat (1976) have indicated the existence of a $15-k p c$ arm.

On the other hand, the galactic longitudes between $l \approx 100^{\circ}$ and $l \approx 140^{\circ}-180^{\circ}$ seems to be deprived of Population I objects (Raharto, 1990). The questions which may arise from these observed phenomena relate to the problem of the metallicity gradient and/or the IMF in the Galaxy. Maeder (1990, this symposium) has clearly indicated the influential factor of metallicity for the formation of WR stars from massive OB stars.

The complication that may arise by direct comparison of the number densities in the solar neighbourhood lies in the inherent nature of the local galactic structure. There are two, perhaps three branches of the local arm within $3 k p c$ from the Sun. The smearing process involved in the determination of number densities may become serious if one compares number densities of elements of galactic volumes of different characteristics. At a particular galactic longitude, the line of sight may largely find interarm regions, while in other directions the line of sight is looking alongside the spiral arm. In different galactic spiral arms, processes which lead to the formation of WR stars may be different because of different IMF and metallicity. The metallicity differences can be divided in two components: arm-to-arm differences as well as galactic radial differences.

We suggest to investigate WR and OB number densities in selected galactic longitude zones in order to discriminate the arm and inter-arm regions. This reduces the statistics however. Within $2.5 \mathrm{kpc}$ from the Sun, $50 \%$ of the WR stars are found in clusters and associations, while of all known galactic WR stars, 44 (i.e. $30 \%$ ) belong to clusters and associations.

\section{The IMF}

This parameter cannot be determined independently. It is based on the observed luminosity function, with evidence of complex variations on spatial, temporal and, lightly, luminosity scales. The commonly accepted law for the luminosity function is that it may vary in a simple parameterized way. Van den Bergh (1986) shows that a fixed IMF can produce different luminosity functions, depending on abundances.

Miller and Scalo (1979) indicated that the combined effect of stellar wind mass loss and binary mass exchange changes the exponential law, which causes the variation of massive stars observed in different regions of the Galaxy.

Because differences in IMF have strong effects on the formation of WR stars, as shown by Arnault et al. (1989) in the case of both star formation bursts and constant formation rates, the use of an observed luminosity function should be taken with care.

\section{Some results}

Assuming the recent finding of Blitz and Spergel (1989) would not pose serious changes to the picture within $2.5 \mathrm{kpc}$ from the Sun, the number ratios of WR and OB stars in some clusters and associations are studied. The galactic regions studied have been chosen on the 
basis of Bok's (1981) local structure.

The lower limits of the initial $O$ star masses have been chosen as $15 \mathrm{M}_{\odot}$ for the WN stars and $35 \mathrm{M}_{\odot}$ for the WC stars, following van der Hucht et al. (1988). Table 1 gives the $\mathrm{WN} / \mathrm{OB}$ number ratios in four regions.

Table 1. WR/OB star number ratios.

\begin{tabular}{|c|c|c|c|c|}
\hline $\mathrm{M}_{O B}$ minimum & Carina & Sag-Sco & Orion & Perseus \\
\hline $15 \mathrm{M}_{\odot}$ & 0.20 & 0.16 & 0.14 & small \\
$35 \mathrm{M}_{\odot}$ & 0.30 & 0.2 & 0.2 & small \\
\hline
\end{tabular}

\section{Conclusion}

If compared to the average WR/OB number ratio in the solar neighbourhood of 0.14 (van der Hucht et al., 1988), the results given above show only marginal differences. Whether those reflect real spatial differences in the luminosity function of massive stars requires deeper study.

\section{References}

Arnault, Ph., Kunth, D., Schild, H. 1989, Astron. Astrophys. 224, 73.

van den Bergh, S. 1986, Astrophys. Space Sci. 118, 435.

Blitz, L., Spergel, D.N. 1989, Bull. American Astron. Soc. 21, 1189.

Bok, B. 1981, H.N. Russell Lecture, Publ. Steward Obs. No. 435.

Conti, P.S., Garmany, C.D., de Loore, C., Vanbeveren, D. 1983, Astrophys. J. 274, 302.

Conti, P.S., Vacca, W.D. 1990, Astron. J. 100, 431.

FitzGerald, M.P., Moffat, A.F.J. 1976, Astron. Astrophys. 50, 149.

Garmany, C.D., Conti, P.S., Chiosi, C. 1982, Astrophys. J. 263, 777.

van der Hucht, K.A., Hidayat, B., Admiranto, A.G., Supelli, K.R., Doom, C. 1988, Astron. Astrophys. 199, 217.

Miller, G.E., Scalo, J.M. 1979, Astrophys. J. Suppl. 41, 533.

Raharto, M. 1990, in: Proc. 5th Asian-Pacific Regional IAU Meeting, Sydney, Australia, in press.

Smith, L.F. 1968, Monthly Notices Roy. Astron. Soc. 141, 317.

\section{DISCUSSION}

Montmerle: How many of these stars belong to $\mathrm{OB}$ associations with giant $H I I$ regions? Hidayat: There are five $\mathrm{OB}$ associations included in these simple statistics.

Conti: Among known galactic WR stars there is only one giant $H I I$ region, NGC 3603, with a WR star. Carina, which has three WR stars, is not quite a giant $H I I$ region by all definitions. 


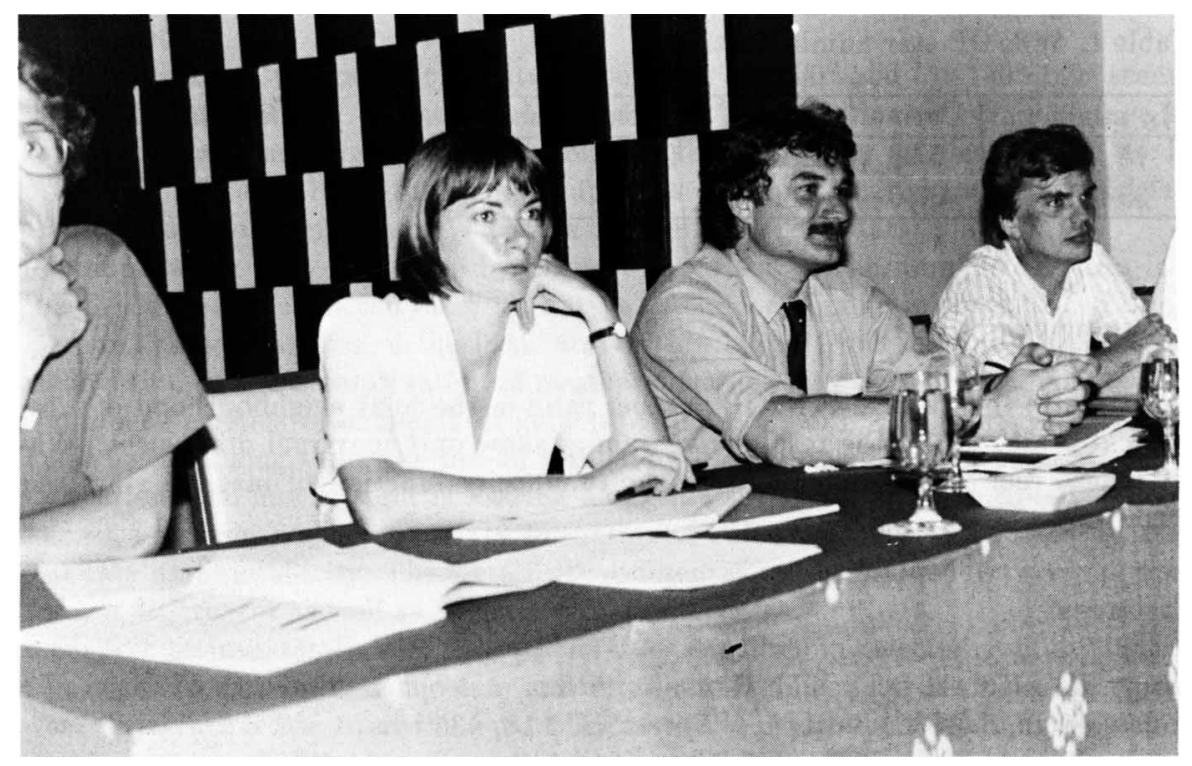

Ian Howarth, Linda Smith, John Hillier, Wolf-Reiner Hamann 